\title{
The taxonomic and phylogenetic relationships of Trypanosoma vivax from South America and Africa
}

\author{
A. P. CORTEZ ${ }^{1}$, R. M. VENTURA ${ }^{1}$, A. C. RODRIGUES ${ }^{1}$, J. S. BATISTA ${ }^{2}$, F. PAIVA ${ }^{3}$, \\ N. ANEZ ${ }^{4}$, R. Z. MACHADO ${ }^{5}$, W. C. GIBSON ${ }^{6}$ and M. M. G. TEIXEIRA ${ }^{1 *}$ \\ ${ }^{1}$ Department of Parasitology, University of São Paulo (USP), São Paulo, SP, Brazil \\ ${ }^{2}$ Department of Pathology, Federal University of Semiarid (UFERSA), RN, Brazil \\ ${ }^{3}$ Department of Veterinary Pathology, Federal University of Mato Grosso do Sul (UFMS), MS, Brazil \\ ${ }^{4}$ Department of Biology, University of Los Andes, Mérida, Venezuela \\ ${ }^{5}$ Department of Animal Pathology, University of the State of São Paulo (UNESP), Faboticabal, SP, Brazil \\ ${ }^{6}$ School of Biological Sciences, University of Bristol, Bristol, UK
}

(Received 16 December 2005; revised 23 February 2006; accepted 25 February 2006; first published online 2 May 2006)

\section{SUMMARY}

The taxonomic and phylogenetic relationships of Trypanosoma vivax are controversial. It is generally suggested that South American, and East and West African isolates could be classified as subspecies or species allied to T. vivax. This is the first phylogenetic study to compare South American isolates (Brazil and Venezuela) with West/East African T. vivax isolates. Phylogeny using ribosomal sequences positioned all $T$. vivax isolates tightly together on the periphery of the clade containing all Salivarian trypanosomes. The same branching of isolates within T. vivax clade was observed in all inferred phylogenies using different data sets of sequences (SSU, SSU plus 5.8S or whole ITS rDNA). T. vivax from Brazil, Venezuela and West Africa (Nigeria) were closely related corroborating the West African origin of South American T. vivax, whereas a large genetic distance separated these isolates from the East African isolate (Kenya) analysed. Brazilian isolates from cattle asymptomatic or showing distinct pathology were highly homogeneous. This study did not disclose significant polymorphism to separate West African and South American isolates into different species/subspecies and indicate that the complexity of T. vivax in Africa and of the whole subgenus Trypanosoma (Duttonella) might be higher than previously believed.

Key words: Trypanosoma vivax, taxonomy, phylogeny, evolution, ribosomal genes, Brazil, genetic diversity, South America, West Africa, East Africa.

\section{INTRODUCTION}

Trypanosoma (Duttonella) vivax is a major livestock pathogen, which is cyclically transmitted between domestic and wild ruminants by tsetse flies over most of its range in Africa. However, it can also be mechanically transmitted by other biting flies, and has therefore been able to spread beyond the African tsetse belt to Central and South America in recent centuries (Gardiner, 1989; Gardiner and Mahmoud, 1992).

Trypanosoma vivax was first reported in the New World in cattle in French Guiana and named as T. guyanense (Leger and Vienne, 1919, cited by Hoare, 1972). Later, renamed $T$. vivax viennei, it was reported in other parts of Central and South America (Hoare, 1972; Shaw and Lainson, 1972). In South America this species has an overlapping distribution with T. evansi (Ventura et al. 2000, 2001). There are confirmed reports of its presence in 10

* Corresponding author: Department of Parasitology, Institute of Biomedical Science, University of São Paulo, São Paulo, SP, 05508-900, Brazil. Fax: +55 1130917417. E-mail:mmgteix@icb.usp.br
South American countries, including Colombia, Venezuela, French Guiana, Bolivia, Peru and Brazil (Jones and Davila, 2001). Shaw and Lainson (1972) reported the first occurrence of $T$. vivax in Brazil, in water buffalo in the Pará State of the Amazon Region. T. vivax outbreaks causing wasting and haematological changes were reported in cattle in Pantanal, a wetland region in Central Brazil and in Bolivia (Silva et al. 1999), but asymptomatic cattle have also been commonly found in Pantanal (Paiva et al. 2000; Ventura et al. 2001). A T. vivax outbreak with severe disease was recently reported in the Paraiba State, a semi-arid region of Northeastern Brazil (Batista et al., manuscript in preparation). Nowadays, T. vivax is commonly found in enzootic equilibrium in the Brazilian Pantanal and surroundings (Ventura et al. 2001; Davila et al. 2003). Whether outbreaks and different disease syndromes are associated with particular T. vivax isolates, or with host factors such as poor health condition or breed remains to be elucidated. Similarly, in Africa $T$. vivax shows variable levels of virulence and distinct pathogenicity. In West Africa, T. vivax infection in cattle is often acute and accompanied by weight loss, reduced milk yields, abortions and 
mortality. In contrast, with the exception of sporadic haemorrhagic syndrome in cattle, East African isolates of $T$. vivax tend to produce mild infections, which are self-limiting in healthy animals (Gardiner and Mahmoud, 1992).

Besides pathogenicity and virulence, $T$. vivax and related taxa have also been reported to differ in morphology and molecular features. Short trypomastigote forms were associated with acute disease in cattle in West Africa and long forms with chronic infection in East Africa. Isolates from Central Africa (Uganda and Congo) classified as T. uniforme presented the smallest forms of the subgenus T. (Duttonella). A caprine trypanosome from East Africa was described as a separated species (T. caprae) and later reclassified as T. vivax ellipsiprymni due its morphological peculiarities. South American and West African isolates, although morphologically indistinguishable, were separated in 2 subspecies, T. vivax vivax and T. vivax viennei, according to cyclical or mechanical transmission, respectively (Hoare, 1972; Shaw and Lainson, 1972; Gardiner and Mahmoud, 1992).

Although relatively few isolates of $T$. vivax have been compared by molecular techniques, all studies revealed differences according to geographical origin. Isoenzyme, satellite DNA, kDNA minicircles and karyotype patterns grouped West African and South American (Colombian) isolates together, and apart from East African (Kenya) isolates (Fasogbon et al. 1990; Dirie et al. $1993 a, b)$, corroborating the hypothesis that T. vivax was introduced into South America with bovines imported from West Africa (Hoare, 1972; Gardiner and Mahmoud, 1992; Dirie et al. $1993 a, b)$. T. vivax from Central Africa shared molecular features with both the East and West African isolates (Fosogbon et al. 1990; Gardiner, 1989).

Studies based on SSU ribosomal RNA (SSU rRNA) have addressed the phylogeny of $T$. vivax and its peculiarities within the genus Trypanosoma (Haag et al. 1998; Stevens and Rambaut, 2001; Stevens et al. 2001). Most phylogenies supported the monophyly of Trypanosoma and positioned $T$. vivax on the clade containing all Salivarian trypanosomes (Stevens and Rambaut, 2001; Stevens et al. 2001; Hamilton et al. 2004; Piontkivska and Hughes, 2005; Suzuki et al. 2005). However, Hughes and Piontkivska (2003) based on phylogenetic analysis done with a larger data set of both outgroup and ingroup taxa than used in previous studies, demonstrated that Salivarian trypanosomes, except for $T$. vivax, formed a highly supported clade outside the clade formed by Stercorarian trypanosomes and other trypanosomatid genera, thus providing strong evidence against the monophyly of Trypanosoma. According to their analysis, the position of T. vivax was not considered resolved and this species clustered apart from all other trypanosomatids outside even bodonids. Nevertheless, all published phylogenies of $T$. vivax to date were based on a single isolate (Y486 from Nigeria), and analyses of more isolates using different genes are urgently required to clear up this question. To assess the genetic diversity and taxonomic position of $T$. vivax in this study we compared SSU, 5.8S and ITS ribosomal sequences aiming (a) to infer phylogenetic relationships among South American (Brazil and Venezuela), West and East African isolates, (b) to compare T. vivax isolates of different regions and from cattle showing distinct clinical and pathological features and (c) to re-examine the taxonomy of the subgenus Trypanosoma (Duttonella) and the validity of the South American subspecies T. vivax viennei.

\section{MATERIALS AND METHODS}

\section{Origin, identification, and clinical features of trypanosomes}

In this study we compared $6 T$. vivax isolates, four from South America, from different outbreaks with cattle showing different clinical and pathological features, and 2 from Africa (Table 1). South American isolates of $T$. vivax were obtained from the blood of naturally infected cattle or from experimentally infected sheep as before (Ventura et al. 2001). Giemsa-stained blood smears from cattle and sheep infected by these isolates were analysed. All these isolates were submitted to a $T$. vivax-specific PCR assay based on spliced-leader gene sequence (Ventura et al. 2001). Details of the African isolates used in this study are as follows: West African T. vivax Y486 from Nigeria (Leeflang et al. 1976) grown in mice and donated by Dr Théo Baltz (University of Bordeaux, France) and clone ILDat 1.2 derived from $T$. vivax Y486; East African isolate IL3905 from Kenya (Rebeski et al. 1999), grown in cell culture and donated by Dr Dierk E. Rebeski (FAO, Austria).

\section{DNA templates, PCR amplification, sequencing and alignment of ribosomal sequences}

Genomic DNA of trypanosomes from blood of cattle or sheep, preserved at $-20{ }^{\circ} \mathrm{C}$ or dried on filter papers, were extracted by phenol-chloroform according to the method reported by Ventura et al. (2001). The oligonucleotides employed for PCR amplifications of ribosomal sequences were described previously (Maia da Silva et al. 2004; Rodrigues et al. 2006). Due to poor quality of DNA templates only the region corresponding to $\mathrm{V} 7-\mathrm{V} 8$ SSU sequences could be amplified for most samples. The amplified products of SSU and whole ITS $($ ITS1 + 5.8S + ITS2) genes were cloned and at least 3 clones from each gene/isolate were sequenced. 
The ribosomal sequences of the SSU, 5.8S and ITS (ITS1 and ITS2) genes of $T$. vivax isolates determined in this study were aligned with sequences from several other trypanosome species using the BioEdit program followed by visual optimization. SSU rRNA sequences from other species of trypanosomes were retrieved from GenBank (Accession number): T. b. brucei (M12676); T. b. gambiense (AJ009141); T. b. rhodesiense (AJ009142); T. congolense Kilifi (AJ009144); T. congolense savannah (AJ009146); T. congolense forest (AJ009145); T. simiae (AJ009162); T. godfreyi (AJ009155); T. equiperdum (AJ009153); T. evansi (D89527); T. sp. D30 from deer (AJ009165); T. theileri Tthc3 from cattle (AY773681); T. theileri Tthb12 from buffalo (AY773678); T. pestanai (AJ009159), T. sp. H26 (AJ009169); T. cruzi Sylvio X10 (AJ009147), T. cruzi CL (AF245383); T. rangeli San Agustin (AJ012417); T. rangeli legeri (AY491769); T. cyclops (AJ250743). Sequences from Bodo caudatus (X53910) and Bodo designis (AF209856) were used as outgroup for Trypanosoma, and sequence of the Parabodonida AT1-3 (AF50051) as outgroup for Trypanosomatidae. We also aligned SSU and ITS sequences retrieved from genome data banks of T. b. brucei, T. congolense and T. vivax Y486 (http:// www.genedb.org). We also analysed an SSU rRNA gene fragment from putative Tanzanian $T$. vivax (AJ563916) (Malele et al. 2003).

\section{Phylogenetic inferences and analysis of GC contents}

Different alignments from distinct data sets were employed in this study. (1) Alignment of SSU ribosomal sequences corresponding to $\mathrm{V} 7-\mathrm{V} 8$ variable region plus conserved flanking region of different species of trypanosomes using bodonids as outgroups (1162 characters). (2) Alignment of these SSU sequences plus $5.8 \mathrm{~S}$ sequences of Salivarian trypanosomes (1169 characters). (3) Alignment of whole ITS (ITS1+5.8S+ITS2 sequences) of T. vivax isolates (554 characters). (4) Alignment of different copies of ITS1 and ITS2 sequences from $T$. vivax, T. b. brucei and T. congolense done separately for each species due to unreliable alignments of these sequences from different species. Maximum parsimony (MP) and maximumlikelihood (ML) analysis were inferred based on V7-V8 and V7-V8 plus 5.8S alignments. The ML model and parameters were estimated using the hierarchical likelihood test implemented in the Modeltest, 3.06 (Posada and Crandall, 1998). MP and ML bootstrapping with 100 replicates were done as before (Hamilton et al. 2004). A dendrogram based on whole ITS sequences (alignment 3) was done using MP. Similarity matrixes were calculated as before (Maia da Silva et al. 2004). Alignments used in this study are available from the authors upon request. Analyses of GC contents were 


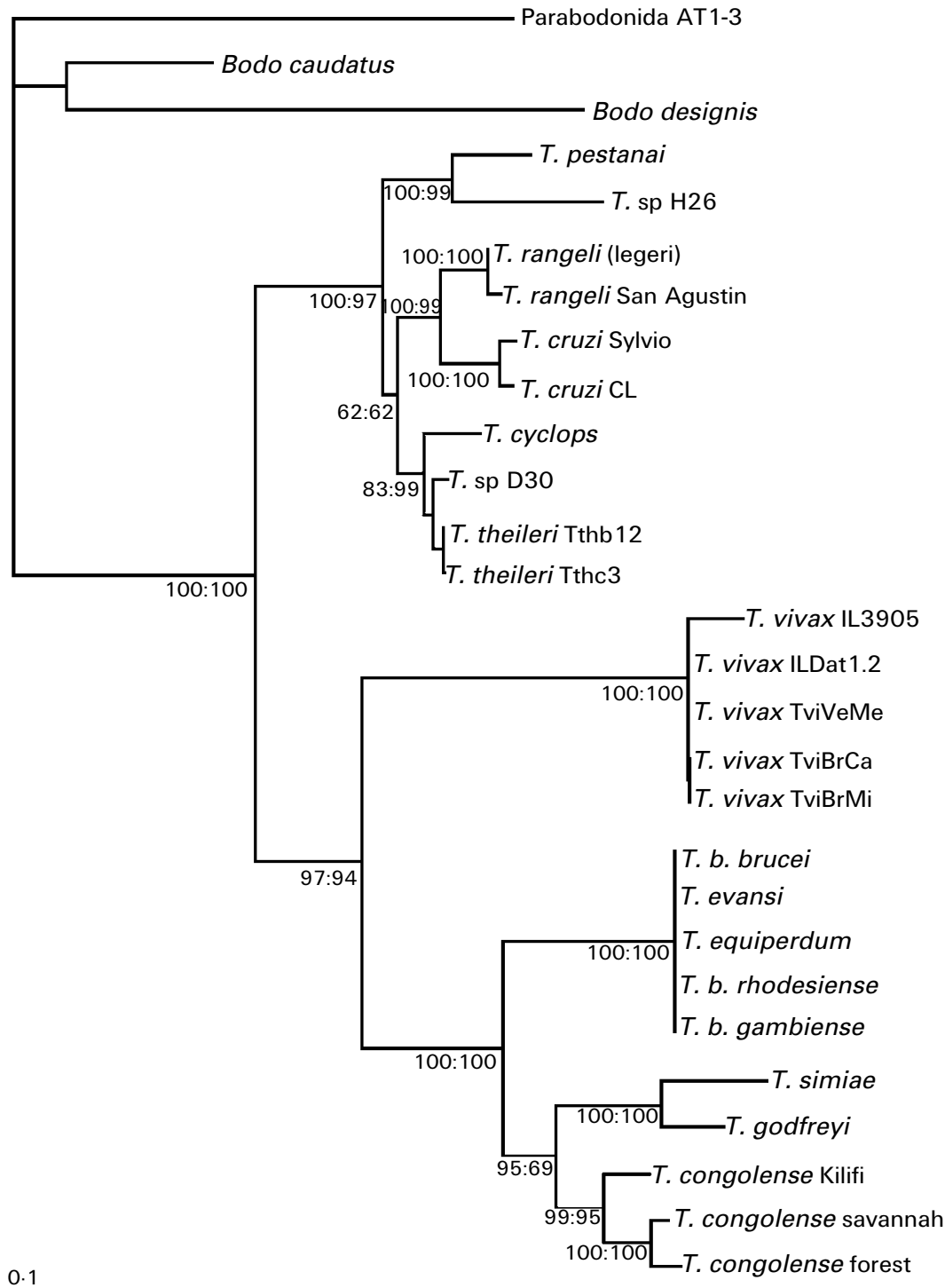

substitutions/site

Fig. 1. Phylogenetic tree based on Maximum Likelihood analysis of SSU (V7-V8) rRNA sequences from Trypanosoma vivax isolates and other trypanosome species. Bodonids (Parabodonida AT1-3, Bodo caudatus, B. designis) were used as outgroup for Trypanosoma. The best-fit evolutionary model for the likelihood analysis (as determined by Modeltest) was Tamura and Nei with gamma and invariant parameters. The numbers at nodes correspond to percentage of bootstrap values (Maximum Parsimony : Maximum Likelihood) derived from 100 replicates.

done using the program MEGA 2.1 (Kumar et al. 2001).

\section{RESULTS}

Identity of T. vivax isolates using morphological and molecular diagnosis

Identification of the South American $T$. vivax isolates (TviBrMi, TviBrPo, TviBrCa and TviVeMe) was originally based on morphology of the trypomastigotes in Giemsa-stained smears of field-collected blood samples from cattle, and subsequently of blood trypanosomes from experimentally infected sheep or calves. All isolates presented similar blood trypomastigotes (Ventura et al. 2001), without significant variability in shape or length, with morphometrical features typical for $T$. vivax from West Africa according to Hoare (1972). Identification of South American isolates was confirmed using a T. vivax specific TviSL-PCR assay (Ventura et al. 2001). This method amplified DNA of all T. vivax isolates from South America and West Africa, whereas DNA from the isolate IL3905 (East Africa) could not be amplified (data not shown).

Phylogeny of T. vivax isolates based on $S S U$ and $5.8 S$ ribosomal sequences

Both ML (Fig. 1) and MP (not shown) trees inferred using SSU rRNA sequences of a diverse range of 
Salivarian and other trypanosome species showed very similar topologies and the same position for all $T$. vivax isolates. T. vivax was always positioned marginally in the clade containing all Salivarian trypanosomes within the genus Trypanosoma. In agreement with this positioning, $T$. vivax divergence was high $(\sim 30 \%)$ when compared with Salivarian species belonging to subgenera $T$. (Trypanozoon) (T. brucei, T. evansi and T. equiperdum) and $T$. (Nannomonas) ( $T$. congolense, $T$. godfreyi and $T$. simiae). In this study we employed as outgroups bodonids and a deep-sea kinetoplastid (Parabodonida) considered to be closer to bodonids than to euglenids (Piontkivska and Hughes, 2005). In analysis including more distantly related euglenid species, the $T$. vivax clade also clustered with Salivarian trypanosomes (99\% bootstrap), and this clade with all other trypanosomes (not shown), although supported by a lower bootstrap value $(76 \%)$. In addition to this study, we previously showed that trees based on V7-V8 SSU rRNA sequences generated a similar branching pattern and all major clades (Maia da Silva et al. 2004; Rodrigues et al. 2006) compared to trees generated using larger SSU rRNA sequences (Stevens et al. 2001; Hamilton et al. 2004).

At least 3 independent SSU rRNA sequences from each South American isolate and from the East African isolate were obtained and compared with sequences from the West African Y486 (genome data bank) and Y486 clone ILDat (GenBank). Brazilian isolates did not show significant sequence polymorphism (average $\sim 0.15 \%$ ) and divergence was only $\sim 0.4 \%$ between these isolates and the Venezuelan isolate. While there was little divergence between West African and South American isolates $(\sim 0.34 \%)$, sequences of the East African isolate were highly divergent compared with all other $T$. vivax isolates $(\sim 3 \cdot 2 \%)$.

A partial SSU rRNA sequence corresponding to variable V7 region amplified directly from tsetse collected in East Africa (Tanzania) and attributed to a $T$. vivax-like trypanosome (Malele et al. 2003) was aligned with corresponding sequences analysed in this study. This sequence was highly divergent from other $T$. vivax sequences, including that of the Kenyan isolate sequenced here $(\sim 11 \%$ sequence divergence). Despite this, phylogenetic analysis clearly clustered this sequence in the $T$. vivax clade (data not shown).

To evaluate the relationships among $T$. vivax isolates and the positioning of these isolates within the clade comprising the Salivarian trypanosomes, we decided to use a combined data set formed by variable (V7-V8) and conserved (5.8S) sequences for phylogenetic analysis. V7-V8 regions are variable sequences flanked and interspersed by conserved regions, and are thus good targets for comparison of related organisms. The $5.8 \mathrm{~S}$ sequences are also good targets allowing very consistent alignments due to conservation among different species of the same subgenus, and significant variability among species belonging to distinct subgenera. We restricted this analysis to Salivarian trypanosomes (all 5.8S sequences obtained in this study plus all available sequences from GenBank), because the alignment of closely related trypanosomes allowed characters in more variable regions to be aligned with higher confidence and included in the ML analysis. The topology of the inferred Salivarian trypanosome tree (Fig. 2) revealed the same branching pattern obtained for these trypanosomes in the phylogeny of Trypanosoma using only V7-V8 SSU rRNA sequences (Fig. 1). The position of the T. vivax clade as a marginal group of the Salivarian clade was confirmed. South American and West African isolates were clustered tightly together, whereas the isolate from East Africa was clearly separated, although closer to other T. vivax isolates than to any other trypanosome species (Fig. 2).

Analysis of genetic relatedness using only the $5.8 \mathrm{~S}$ sequences also separated the East African T. vivax isolate from the group formed by South American and West African isolates. Comparison of aligned $5.8 \mathrm{~S}$ sequences from different clones of South American T. vivax isolates showed a divergence varying from $\sim 0.6 \%$, among Brazilian isolates, to $\sim 1 \cdot 2 \%$, between Brazilian and Venezuelan isolates. Sequence divergence between South American and African isolates ranged from $\sim 0.7 \%$ to $\sim 3.2 \%$ for West and East African isolates, respectively. Contrasting with the highly conserved $5.8 \mathrm{~S}$ sequences of American and West African isolates ( $\sim 0.4 \%$ divergence), there is a large polymorphism $(\sim 1 \cdot 0 \%)$ among sequences of 8 clones of $5.8 \mathrm{~S}$ from the East African isolate IL3905. Analysis of $5.8 \mathrm{~S}$ gene sequences from data banks disclosed high sequence conservation (minimum $99 \cdot 8 \%$ ) in T. brucei, $T$. congolense and T. vivax Y486 ILDat 1.2. Divergence separating $5.8 \mathrm{~S}$ sequences of $T$. vivax from T. congolense or $T$. brucei was $\sim 15 \%$ and $\sim 16 \%$, respectively.

\section{Polymorphism and genetic relatedness among \\ T. vivax isolates based on ITS rDNA sequences}

To demonstrate the genetic diversity within the T. vivax clade, we also evaluated polymorphisms among 6 isolates examined in this study using the more divergent ITS region of the rRNA gene array. For analysis of ITS polymorphism we compared Brazilian isolates from distant geographical regions (Central and Northeast), recovered from cattle showing different clinical and pathological profiles (Table 1) with isolates from Venezuela and Africa. We investigated the polymorphism by comparing length and sequence of the PCR-amplified DNA fragments containing the whole ITS rDNA (ITS1, 


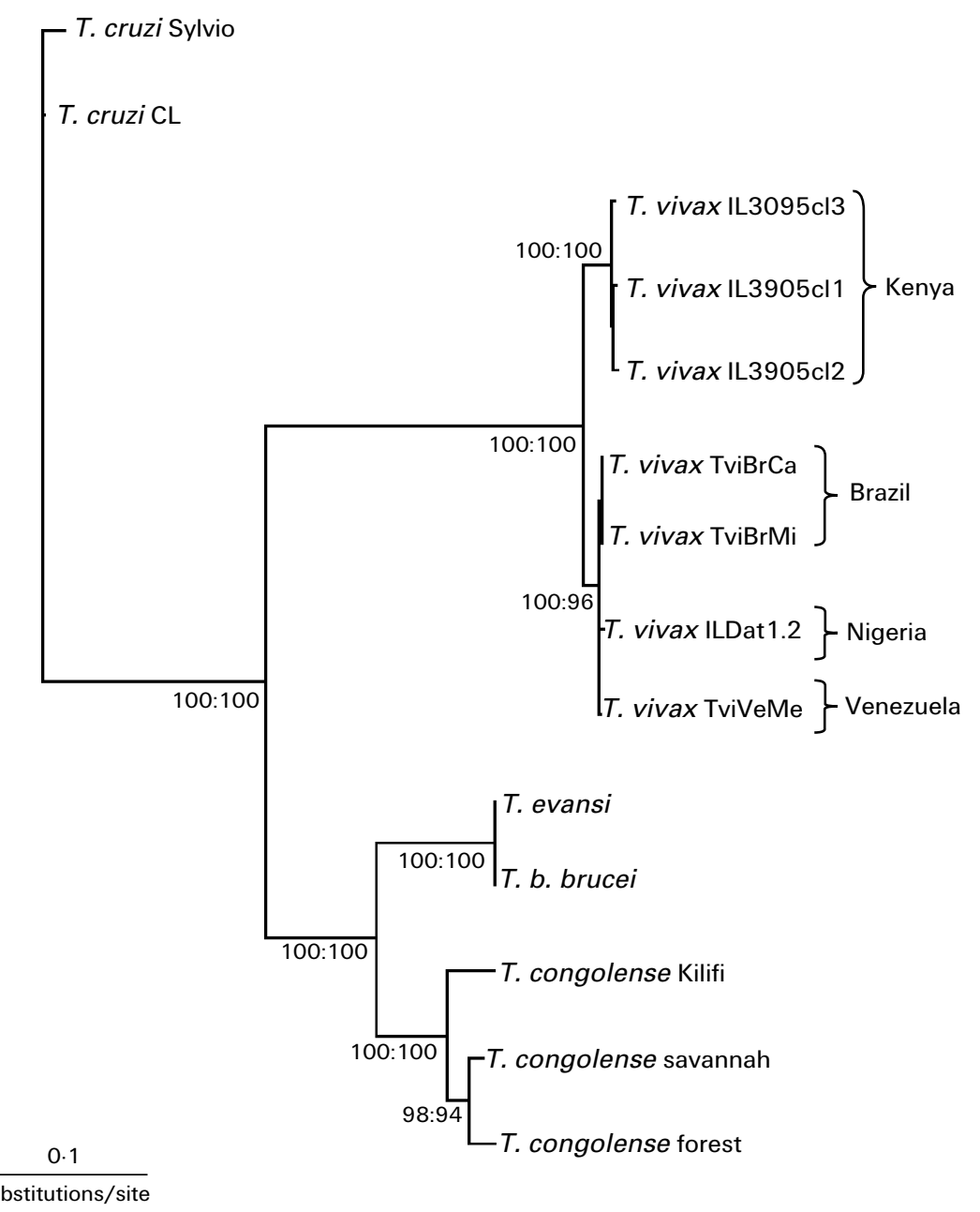

Fig. 2. Phylogenetic tree based on Maximum Likelihood analysis of SSU and ITS (V7-V8 + 5.8S) rDNA sequences from Trypanosoma vivax isolates and other salivarian trypanosome species. The best-fit evolutionary model for the likelihood analysis (as determined by Modeltest) was Tamura and Nei with gamma. The numbers at nodes correspond to percentage of bootstrap values (Maximum Parsimony : Maximum Likelihood) derived from 100 replicates.

5.8S and ITS2) sequences. All South American/ West African isolates had sequences of similar length (490 bp). However, the East African isolate IL3905 had different ITS lengths, varying from 525 to $534 \mathrm{bp}$. Small ITS sequence divergence separated American and West African isolates ( 0.8\%). However, a high polymorphism separated $T$. vivax of South America/West Africa from the East African IL3905 isolate, with the average divergence index increasing drastically to $\sim 33 \%$. We selected the 2 most polymorphic cloned sequences of ITS $($ ITS1 + 5.8S + ITS2) from each isolate to be included in the alignment used to assess genetic relatedness among $T$. vivax isolates, with the exception of the isolate IL3905, for which ITS sequences of 8 clones were included to represent the high degree of polymorphism within this isolate (Fig. 3). Other trypanosome species were not included in the alignment, because their ITS sequences could not be aligned with confidence. Despite the low degree of genetic variability, all ITS sequences from South American isolates were always grouped with those from West African isolates in a relatively homogeneous cluster, segregated in unsupported subclusters (Fig. 3). This cluster was clearly separated from that formed by the heterogeneous sequences from the East African isolate (Fig. 3), corroborating the segregation pattern of $T$. vivax isolates based on more conserved SSU and 5.8S ribosomal sequences (Figs 1 and 2).

The genetic polymorphism detected among all T. vivax isolates, in both ITS1 ( 27\%) and ITS2 ( $\sim 25 \%)$ sequences, comprises several large blocks of deletions and insertions, in addition to numerous substitutions. Alignment of ITS2 sequences of all $T$. vivax isolates illustrates the polymorphism within the same isolate and among isolates of this species (Fig. 4). Analyses of sequence polymorphism among 3-4 clones from each $T$. vivax isolate showed low ITS1 and ITS2 divergence among sequences from Brazilian and Venezuelan isolates $(\sim 0.6 \%$ for both ITS1 and ITS2). However, analysis of ITS sequences of $T$. vivax Y486 revealed a significant polymorphism $(\sim 2.7 \%$ and $1.6 \%$ for ITS1 and 


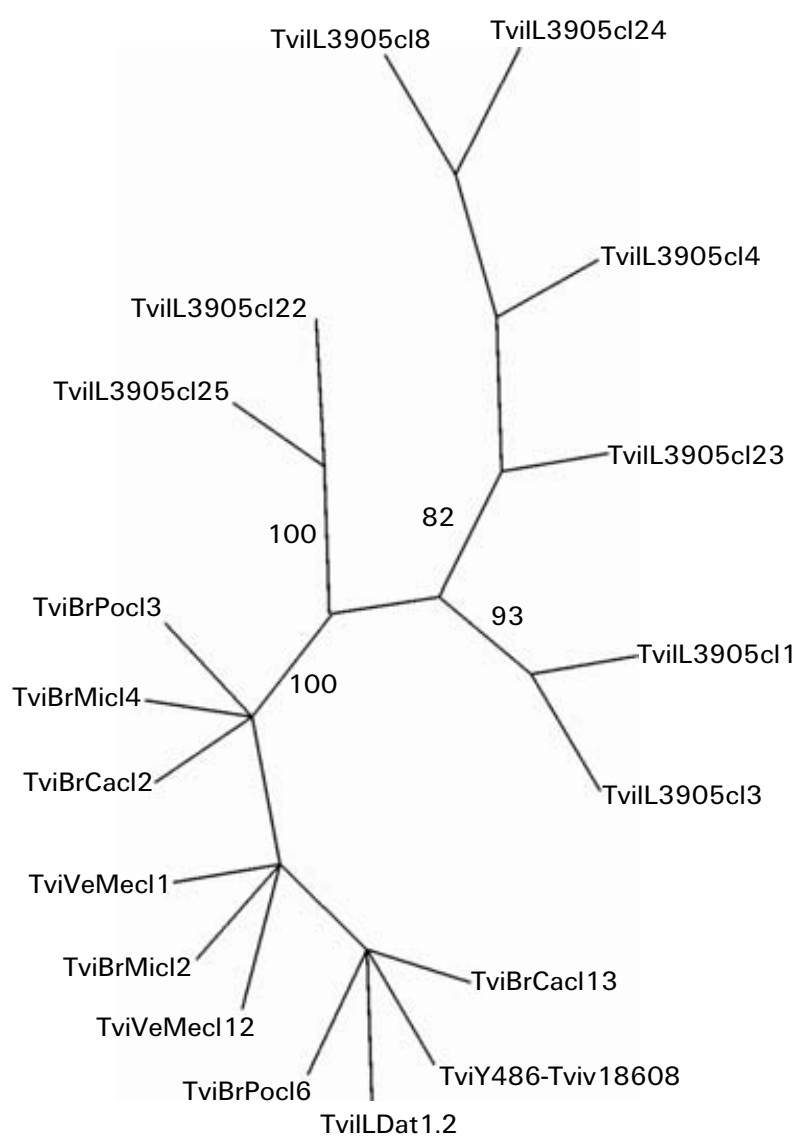

Fig. 3. Unrooted tree based on Parsimony analysis of whole ribosomal ITS sequences (ITS1 + 5.8S + ITS2) from Trypanosoma vivax isolates. The numbers at nodes correspond to percentage of bootstrap values derived from 100 replicates.

ITS2, respectively). The highest polymorphism was observed among the ITS sequences of IL3905, varying from $\sim 1.0$ to $8.7 \%$ of divergence (average of $\sim 7 \cdot 0 \%$ and $6 \cdot 8 \%$ for ITS1 and ITS2, respectively). Analysis of ITS sequences from data banks disclosed a high polymorphism within $T$. congolense savannah (maximum $\sim 9.7 \%$ for ITS1 and $\sim 8.6 \%$ for ITS2), whereas $T$. b. brucei showed more conserved sequences, although still significantly polymorphic ( $2 \cdot 8 \%$ for ITS1 and $1 \cdot 2 \%$ for ITS2).

\section{Analysis of $G C$ contents in the ribosomal genes of T. vivax isolates}

Previous studies showed that the whole SSU rDNA of T. vivax Y486 has a high GC content when compared with several other trypanosome species, with $\sim 55.4 \%$ GC, which is $\sim 3.0 \%$ higher than any other trypanosomatid (Haag et al. 1998; Stevens and Rambaut, 2001). To verify if the high GC content is shared by all $T$. vivax isolates analysed in this study, we compared different regions of rRNA genes of these organisms and other trypanosome species. Results showed that the GC contents of sequences from V7-V8 SSU rDNA are quite similar among American and West African isolates of $T$. $\operatorname{vivax}(\sim 63 \%)$. The $\mathrm{GC}$ content of sequences of the East African isolate was in the same range ( $\sim 64 \%$ ), despite the high genetic distance separating these isolates. Comparison with other Salivarian trypanosomes showed that the average GC content of the V7-V8 SSU rDNA region of $T$. vivax isolates was significantly higher than other subgenera (subgenus T. (Trypanozoon) $\sim 52 \%$, subgenus T. (Nannomonas) $\sim 56 \%$ ). Similarly, non-salivarian trypanosomes e.g. T. cruzi, T. rangeli, T. theileri and other species included in Fig. 1 also showed lower GC values $(\sim 52 \%)$. High GC contents were also observed in the $5.8 \mathrm{~S}$ sequences of $T$. vivax isolates compared to other trypanosomes: T. vivax 57-58\%; subgenus T. (Trypanozoon) $\sim 49 \%$; subgenus T. (Nannomonas) $\sim 50 \%$. Thus, so far, high GC content in ribosomal sequences of mammalian trypanosomes appears to be unique to $T$. vivax.

\section{DISCUSSION}

In this study we compared South American and African isolates of $T$. vivax by phylogenetic analysis based on a data set consisting of conserved (5.8S) and variable (V7-V8 regions of SSU rRNA) ribosomal sequences. All T. vivax isolates clustered tightly together on the margin of the clade containing all Salivarian trypanosomes (clade T. brucei), in agreement with previous studies (Stevens et al. 2001; Stevens and Rambaut, 2001; Hamilton et al. 2004; Piontkivska and Hughes, 2005). Based on SSU rDNA phylogenies, the Salivarian trypanosomes seem to be the most rapidly evolving group of trypanosomes, with $T$. vivax evolving faster than all other Salivarian species and the entire range of trypanosomatids (Stevens and Rambaut, 2001). In this study we demonstrated that all T. vivax isolates analysed share this high evolutionary rate and have higher GC contents of ribosomal sequences than all other trypanosomatids, as previously shown for T. vivax Y486 (Haag et al. 1998; Stevens and Rambaut, 2001).

The position of T. vivax in the phylogenetic trees suggests that it was the first taxon to diverge, thus representing the most ancient species of Salivarian trypanosome (Haag et al. 1998; Stevens et al. 2001; Stevens and Rambaut, 2001; Hamilton et al. 2004). Although all Salivarian trypanosomes are transmitted via tsetse saliva, the life-cycle of $T$. vivax is distinct in that it does not undergo development in the fly hindgut like the other subgenera, but completes its development wholly within the mouthparts (Hoare, 1972). In common with the other Salivarian trypanosomes, T. vivax undergoes antigenic variation, but shows distinct features, e.g. biochemical and antigenic properties of the variant surface glycoproteins (VSGs) and smaller and more heterogeneous VAT (variable antigen type) 


\begin{tabular}{|c|c|}
\hline $\begin{array}{l}3905 \mathrm{c} 13 \\
3905 \mathrm{cl1}\end{array}$ & 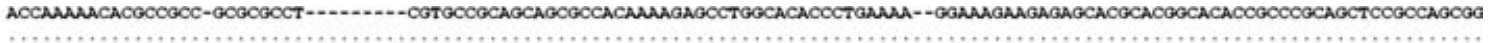 \\
\hline $23905 \mathrm{e} 14$ & \\
\hline 3905018 & \\
\hline$-3905 \mathrm{cl} 24$ & \\
\hline 3 & \\
\hline IL.3905e125 & …na \\
\hline IL $3905 \mathrm{cl} 22$ & . \\
\hline $\mathrm{vin}$ & ...A...A. TOCOCACTGCA. \\
\hline visec & ... CA. A. TOCOCACTOCA. \\
\hline viveHe12 & CA . A TOCOCACCOCA. \\
\hline W & .... СА...A. тOCGCACTGCA. \\
\hline & .... CA. A. TOCOCACTGCA. \\
\hline TViBrpo6 & 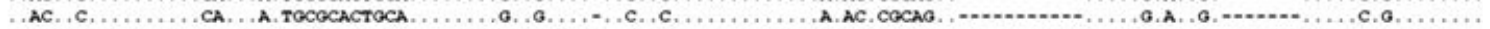 \\
\hline TVil & ... CA...A. TOCOCACTGCA. \\
\hline TV1 & ... CA. A. TOCOCACTOCA. \\
\hline virit & AC. C. ........ CA . A. TOCOCACTOCA. . \\
\hline rviv186bos & 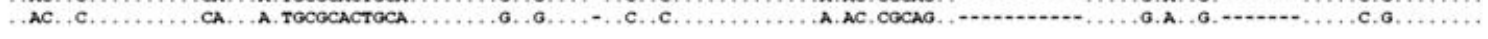 \\
\hline & ATGCACOCACGCCTCTOCAC----GTOCACACACOTOT---ACOCATOCTOCACOCACOCACA-QCA \\
\hline IL3 & \\
\hline & ש \\
\hline IL 3 & T. . \\
\hline IL 3 & $.239 \mathrm{pl}$ \\
\hline IL 3 & A. A.......... TGT. \\
\hline & ............ \\
\hline IL3 & $\therefore \infty$ \\
\hline & AA GAGG A. OG . G.C. \\
\hline TVil & AA, GAGG A, CG , G.C, \\
\hline & 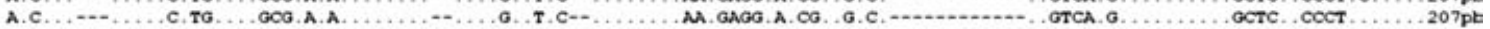 \\
\hline & AA GAGG A. OG G.C. GACA G. \\
\hline & 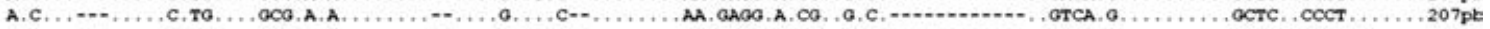 \\
\hline $\mathrm{Nin}$ & AA GAGG A. OG . G.C. \\
\hline & AA GAGOA.CO . G.C. \\
\hline & AA GAGQ A. CQ . G.C. . . \\
\hline 1. & ,.с., \\
\hline & AA GAGG A CO G.C. GTCA $a$. \\
\hline
\end{tabular}

Fig. 4. Alignment of selected ITS2 rDNA cloned sequences of Trypanosoma vivax isolates from different geographical regions.

repertoire (Gardiner et al. 1996). The chromosomal organization in T. vivax is also distinct, since it has only 1-2 minichromosomes contrasting with 50-100 in the other Salivarian trypanosomes (Dickin and Gibson, 1989). T. vivax is predominantly a parasite of bovids, presenting a smaller range of mammalian hosts compared to $T$. congolense or T. brucei (Hoare, 1972; Gibson, 2002). Thus the position of $T$. vivax on the periphery of the T. bruce clade is compatible with its distinct biological and molecular features compared to the rest of the Salivarian trypanosomes.

This study confirmed the close genetic relatedness of $T$. vivax isolates from South America and West Africa (Fasogbon et al. 1990; Dirie et al. $1993 a, b$; Ventura et al. 2001). A large genetic distance separated these isolates from the only East African isolate analysed in this study. These data are in agreement with previous studies that distinguished these two populations of $T$. vivax by morphology (Hoare, 1972); development in tsetse (Moloo et al. 1987); susceptibility of host species and pathology (Leeflang et al. 1976; Gathuo et al. 1987; Williams et al. 1992), besides immunological (Vos and Gardiner, 1990) and molecular (Fasogbon et al. 1990; Dirie et al. $1993 a, b)$ features.

Contrasting with the great genetic distance separating East African T. vivax from all other isolates of this species, a high homogeneity was observed among South American isolates. However, the Brazilian isolates were more closely related to each other than to the Venezuelan isolate. This genetically homogeneous group of Brazilian isolates of T. vivax originated from cattle of distinct outbreaks of disease and was also recovered from asymptomatic cattle.
For example, isolate TviBrPo came from an outbreak in Poconé, Northern Pantanal, Central Brazil, with cattle showing severe haematological changes, mainly anaemia and leucopenia, progressive weakness, marked weight loss and abortions (Silva et al. 1999), while isolate TviBrCa came from an outbreak in Catolé da Rocha, Northeast Brazil, where cattle showed similar symptoms but also presented nervous signs (meningoencephalitis and malacia) with an invariably fatal outcome irrespective of drug treatment (Batista et al. manuscript in preparation). The isolates TviBrMi from Brazil (Paiva et al. 2000) and TviVeMe from Venezuela (Añez et al. manuscript in preparation) were both from chronic and asymptomatic infected cattle. Thus, this study demonstrated that cattle infected with the same or very similar isolates of $T$. vivax can show distinct diseases or be totally asymptomatic.

Very few isolates of $T$. vivax have been analysed from East Africa and thus little is known about genetic variation of isolates from this region. Lack of detection of Kenyan T. vivax using PCR primers derived from a West African isolate indicates the widespread occurrence of genetic variants of this species (Njiru et al. 2004), a fact corroborated in this study by the failure of the PCR based on SL gene sequences of South American/West African T. vivax (Ventura et al. 2001) to detect the East African isolate examined in this study. Other distinct genetic variants of $T$. vivax may well exist, for example that represented by a partial SSU rRNA sequence amplified directly from tsetse collected in Tanzania, East Africa (Malele et al. 2003). The SSU rRNA sequence fragment shows this trypanosome to be more related to $T$. vivax than to any other 
trypanosome species, although significantly divergent from all other T. vivax isolates, including the isolate from Kenya included in this study. Previous studies showed that T. vivax populations in East Africa differed in morphology, host susceptibility, isoenzyme patterns and virulence (Hoare, 1972; Murray and Clarkson, 1982; Gathuo et al. 1987; Fasogbon et al. 1990).

The ability to develop in tsetse flies would not appear to be a major evolutionary factor in this segregation pattern, since West African and South American $T$. vivax are very closely genetically related. Despite considerable divergence among copies of ITS ribosomal genes, especially within the East African isolate, sequences from this isolate were always clustered together in all analyses, clearly separated from sequences of West African and South American isolates, which were also always clustered together. Interestingly, South American isolates had highly homogeneous copies of the ITS sequence compared to the tsetse-transmitted $T$. vivax from Africa. We also detected highly polymorphic ITS regions in $T$. congolense savannah and significant divergence among ITS copies of $T . b$ brucei, which are cyclically transmitted by tsetse, whereas polymorphism was not observed in a mechanically transmitted Brazilian isolate of $T$. evansi (data not shown). Comparison of a larger number of trypanosome isolates from tsetse-infested and tsetse-free regions is required to clarify this evolutionary pattern of ribosomal genes. Our previous studies disclosed small polymorphisms among cloned copies of SSU and ITS sequences among $T$. rangeli (Maia da Silva et al. 2004) and $T$. theileri (Rodrigues et al. 2006) isolates, which are Stercorarian trypanosomes cyclically transmitted by triatomine bugs or tabanids, respectively.

Although $T$. vivax populations from South America and West Africa have different modes of transmission (cyclical or mechanical), they have similar pathology and, as shown by this study, cannot be distinguished clearly by molecular phylogenetic analysis using ribosomal sequences. These data corroborated our previous study based on SL gene sequences (Ventura et al. 2001). South American isolates of $T$. vivax have previously been classified as a distinct subspecies, T. vivax viennei, the validity of which can now be seen to rest on mode of transmission. Since there is widespread suspicion that $T$. vivax can be mechanically transmitted in areas of Africa where tsetse are sparse (Gardiner, 1989; Gardiner and Mahmoud, 1992), a definition of $T$. vivax viennei based only on mode of transmission is clearly problematic. So far, West African and South American (Colombia) isolates of T. vivax have only been found to differ in antigenic profiles (Murray and Clarkson, 1982; Dirie et al. 1993b). In contrast, there would appear to be far greater justification for recognition of the East African forms of $T$. vivax as separate species or subspecies of the subgenus T. (Duttonella). However, this must await the molecular and phylogenetic analysis of greater numbers of $T$. vivax isolates from East Africa.

We are grateful to several collaborators for blood samples of $T$. vivax naturally or experimentally infected animals, for DNA samples and for inestimable help in the fieldwork. We thank the Sanger Centre Institute for making sequences from trypanosome genomes freely available for analysis. This study was sponsored by grants of Brazilian agencies FAPESP and $\mathrm{CNPq}$ given to Marta M. G. Teixeira, and by Venezuelan grant (CDCHT-ULAC1210) given to Nestor Añez. Rogeria M. Ventura is presently at the FMU University. Alane P. Cortez and Adriana C. Rodrigues are CNPq student fellows.

\section{REFERENCES}

Davila, A. M., Herrera, H. M., Schlebinger, T., Souza, S. S. and Traub-Cseko, Y. M. (2003). Using PCR for unravelling the cryptic epizootiology of livestock trypanosomosis in the Pantanal. Veterinary Parasitology 117, 1-13. DOI :10.1016/j.vetpar.2003.08.002.

Dickin, S. K. and Gibson, W. C. (1989). Hybridization with a repetitive DNA probe reveals the presence of small chromosomes in Trypanosoma vivax. Molecular and Biochemical Parasitology 33, 135-142.

DOI :10.1016/0166-6851(89)90027-3.

Dirie, M. F., Murphy, N. B. and Gardiner, P. R. (1993a). DNA fingerprinting of Trypanosoma vivax isolates rapidly identifies intraspecific relationships. The Fournal of Eukaryotic Microbiology 40, 132-134.

Dirie, M. F., Otte, M. J., Thatthi, R. and Gardner, P. R. (1993b). Comparative studies of Trypanosoma (Duttonella) vivax isolates from Colombia. Parasitolology 106, 21-29.

Fasogbon, A. I., Knowles, G. and Gardiner, P. R. (1990). A comparison of the isoenzymes of Trypanosoma (Duttonella) vivax isolates from East and West Africa. International Fournal for Parasitology 20, 389-394.

Gardiner, P. R. (1989). Recent studies of the biology of Trypanosoma vivax. Advances in Parasitology 28, 229-317.

Gardiner, P. R. and Mahmoud, M. M. (1992). Salivarian trypanosomes causing disease in livestock outside sub-saharan Africa. In Parasitic Protozoa (ed. Kreier, J. P. and Baker, J. R.), pp. 277-313. Academic Press, London.

Gardiner, P. R., Nene, V., Barry, M. M., Thatthi, R., Burleigh, B. and Clarke, M. W. (1996). Characterization of a small variable surface glycoprotein from Trypanosoma vivax. Molecular and Biochemical Parasitology 82, 1-11. DOI:10.1016/01666851(96)02687-4.

Gathuo, H. K., Nantulya, V. M. and Gardiner, P. R. (1987). Trypanosoma vivax: adaptation of two East African stocks to laboratory rodents. Fournal of Protozoology 34, 48-53.

Gibson, W. (2002). Epidemiology and diagnosis of African trypanosomiasis using DNA probes. Transactions of 
the Royal Society of Tropical Medicine and Hygiene $\mathbf{9 6}$ S141-S143.

Haag, J., O'Huigin, C. and Overath, P. (1998). The molecular phylogeny of trypanosomes: evidence for an early divergence of the Salivaria. Molecular and Biochemical Parasitology 91, 37-49. DOI:10.1016/ S0166-6851(97)00185-0.

Hamilton, P. B., Stevens, J. R., Gaunt, M. W., Gidley, J. and Gibson, W. C. (2004). Trypanosomes are monophyletic: evidence from genes for glyceraldehyde phosphate dehydrogenase and small subunit ribosomal RNA. International Fournal for Parasitology 34, 1393-1404. DOI : 10.1016/j.ijpara.2004.08.011.

Hoare, C. A. (1972). The Trypanosomes of Mammals. Blackwell Scientific Publications, Oxford.

Hughes, A. L. and Piontkivska, H. (2003). Phylogeny of Trypanosomatidae and Bodonidae (Kinetoplastida) based on 18S rRNA: evidence for paraphyly of Trypanosoma and six other genera. Molecular Biology and Evolution 20, 644-652. DOI : 10.1093/molbev/ msg062.

Jones, T. W. and Davila, A. M. (2001). Trypanosoma vivax-out of Africa. Trends in Parasitology 17, 99-101. DOI :10.1016/S1471-4922(00)01777-3.

Kumar, S., Tamura, K., Jakobsen, I. B. and Nei, M. (2001). MEGA2: molecular evolutionary genetics analysis software. Bioinformatics 17, 1244-1245.

Leeflang, P., Ige, K. and Olatunde, D. S. (1976). Studies on Trypanosoma vivax: the infectivity of cyclically and mechanically transmitted ruminant infections for mice and rats. International Fournal for Parasitology 6, 453-456. DOI:10.1016/00207519(76)90081-3.

Maia da Silva, F. M., Noyes, H., Campaner, M., Junqueira, A. C., Coura, J. R., Anez, N., Shaw, J. J., Stevens, J. R. and Teixeira, M. M. G. (2004). Phylogeny, taxonomy and grouping of Trypanosoma rangeli isolates from man, triatomines and sylvatic mammals from widespread geographical origin based on SSU and ITS ribosomal sequences. Parasitology 129, 549-561. DOI:10.1017/ S0031182004005931.

Malele, I., Craske, L., Knight, C., Ferris, V., Njiru, Z., Hamilton, P., Lehane, S., Lehane, $M$. and Gibson, W. C. (2003). The use of specific and generic primers to identify trypanosome infections of wild tsetse flies in Tanzania by PCR. Infection, Genetics and Evolution 3, 271-279. DOI:10.1016/S15671348(03)00090-X.

Moloo, S. K., Kutuza, S. B. and Desai, J. (1987). Comparative study on the infection rates of different Glossina species for East and West African Trypanosoma vivax stocks. Parasitology 95, 537-542.

Murray, A. K. and Clarkson, M. J. (1982). Characterization of stocks of Trypanosoma vivax. II. Immunological studies. Annals of Tropical Medicine and Parasitology 76, 283-292.

Njiru, Z. K., Makumi, J. N., Okoth, S., Ndungu, J. M. and Gibson, W. C. (2004). Identification of trypanosomes in Glossina pallidipes and G. longipennis in Kenya. Infection, Genetics and Evolution 4, 29-35. DOI:10.1016/j.meegid.2003.11.004.

Paiva, F., De Lemos, R. A. A., Nakazato, L., Mori, A. E., Brum, K. E. and Bernardo, K. C. A. (2000).
Trypanosoma vivax em bovinos no Pantanal do Mato Grosso do Sul, Brasil: I - Acompanhamento clínico, laboratorial e anatomopatológico de rebanhos infectados. Revista Brasileira de Parasitologia Veteterinária 9, 135-141.

Piontkivska, H. and Hughes, A. L. (2005). Environmental kinetoplastid-like 18S rRNA sequences and phylogenetic relationships among Trypanosomatidae: Paraphyly of the genus Trypanosoma. Molecular and Biochemical Parasitology 144, 94-99. DOI:10.1016/j.molbiopara.2005.08.007.

Posada, D. and Crandall, K. A. (1998). MODELTEST: testing the model of DNA substitution. Bioinformatics 14, 817-818.

Rebeski, D. E., Winger, E. M., Van Rooij, E. M. A., Schöchl, R., Schulle, W., Dwinger, R. H., Crowther, J. R. and Wright, P. (1999). Pitfalls in the application of enzyme-linked immunoassays for the detection of circulating trypanosomal antigens in serum samples. Parasitology Research 85, 550-556. DOI:10.1007/s004360050594.

Rodrigues, A. C., Paiva, F., Campaner, M., Stevens, J. R., Noyes, H. A. and Teixeira, M. M. G. (2006). Phylogeny of Trypanosoma (Megatrypanum) theileri and related trypanosomes reveals lineages of isolates associated with artiodactyl hosts diverging on SSU and ITS ribosomal sequences. Parasitology 3, 1-10. DOI : $10.1017 /$ S0031182005008929.

Shaw, J. J. and Lainson, R. (1972). Trypanosoma vivax in Brazil. Annals of Tropical Medicine and Parasitology 66, 25-32.

Silva, R. A., Ramirez, L., Souza, S. S., Ortiz, A. G., Pereira, S. R. and Dávila, A. M. (1999). Hematology of natural bovine trypanosomosis in the Brazilian Pantanal and Bolivian wetlands. Veterinary Parasitology 85, 87-93.

Stevens, J. R. and Rambaut, A. (2001). Evolutionary rate differences in trypanosomes. Infection, Genetics and Evolution 1, 143-150. DOI :10.1016/S15671348(01)00018-1.

Stevens, J. R., Noyes, H. A., Schofield, C. J. and Gibson, W. C. (2001). The molecular evolution of Trypanosomatidae. Advances in Parasitology 48, $1-56$.

Suzuki, T., Hashimoto, T., Yabu, Y., Majiwa, P., Ohshima, S., Suzuki, M., Lu, S., Hato, M., Kido, Y., Sakamoto, K., Nakamura, K., Kita, K. and Ohta, N. (2005). Alternative oxidase (AOX) genes of African trypanosomes: phylogeny and evolution of AOX and plastid terminal oxidase families. The Fournal of Eukaryotic Microbiology 52, 374-381. DOI:10.1111/ j.1550-7408.2005.00050.x.

Ventura, R. M., Takata, C. S., Silva, R. A., Nunes, V. L., Takeda, G. F. and Teixeira, M. M. G. (2000). Molecular and morphological studies of Brazilian Trypanosoma evansi stocks: the total absence of $\mathrm{kDNA}$ in trypanosomes from both laboratory stocks and naturally infected domestic and wild mammals. Fournal of Parasitology 86, 1289-1298.

Ventura, R. M., Paiva, F., Silva, R. A., Takeda, G. F., Buck, G. A. and Teixeira, M. M. G. (2001) Trypanosoma vivax: characterization of the splicedleader gene of a Brazilian stock and species-specific detection by PCR amplification of an intergenic spacer 
sequence. Experimental Parasitology 99, 37-48.

DOI :10.1006/expr.2001.4641.

Vos, G. J. and Gardiner, P. R. (1990). Antigenic relatedness of stocks and clones of Trypanosoma vivax from east and west Africa. Parasitology 100, 101-106.
Williams, D. J., Logan-Henfrey, L. L., Authie, E., Seely, C. and McOdimba, F. (1992). Experimental infection with a haemorrhage-causing Trypanosoma vivax in N'Dama and Boran cattle. Scandinavian Fournal of Immunology 11, 34-36. 